\title{
OPERATOR-WEIGHTED COMPOSITION OPERATORS BETWEEN WEIGHTED SPACES OF VECTOR-VALUED ANALYTIC FUNCTIONS
}

\author{
José Bonet, M. Carmen Gómez-Collado, David Jornet and Elke Wolf
}

Universidad Politécnica de Valencia, Instituto Universitario de Matemática Pura y Aplicada Edificio 8E, Cubo F, 4ª Planta, E-46022 Valencia, Spain; jbonet@mat.upv.es

Universidad Politécnica de Valencia, Instituto Universitario de Matemática Pura y Aplicada Edificio 8E, Cubo F, 4ª Planta, E-46022 Valencia, Spain; mcgomez@mat.upv.es

Universidad Politécnica de Valencia, Instituto Universitario de Matemática Pura y Aplicada Edificio 8E, Cubo F, 4ª Planta, E-46022 Valencia, Spain; djornet@mat.upv.es

University of Paderborn, Mathematical Institute

Warburger Str. 100, D-33098 Paderborn, Germany; lichte@math.uni-paderborn.de

\begin{abstract}
We investigate several properties of operator-weighted composition maps $W_{\psi, \varphi}: f \mapsto$ $\psi(f \circ \varphi)$ on unweighted $H(\mathbf{D}, X)$ and weighted $H_{v}^{\infty}(\mathbf{D}, X)$ spaces of vector-valued analytic functions on the unit disc D. Here $\varphi$ is an analytic self-map of $\mathbf{D}$ and $\psi$ is an analytic operator-valued function on $\mathbf{D}$. We characterize when the operator is continuous, maps a neighbourhood into a bounded set or maps bounded sets into relatively compact sets. In this way we extend results due to Laitila and Tylli for the case of Banach valued functions. This more general setting permits us to compare the results in the unweighted and weighted cases. New examples are provided, especially when the spaces $X$ and $Y$ are Köthe echelon spaces. They show the differences between the present setting and the case of functions taking values in Banach spaces.
\end{abstract}

\section{Introduction and preliminaries}

The purpose of this paper is to investigate qualitative properties of operatorweighted composition operators on spaces of vector-valued analytic functions. To describe the content of the paper we need some notation. If $X$ is a Hausdorff locally convex space, $H(\mathbf{D}, X)$ denotes the space of all analytic functions $f: \mathbf{D} \rightarrow X$. If $X=\mathbf{C}$ we simply write $H(\mathbf{D})$. This space is endowed with the topology of uniform convergence on the compact subsets of $\mathbf{D}$; which makes $H(\mathbf{D})$ a (nuclear) Fréchet space. Our notation for locally convex spaces is standard and we refer the reader to $[14,15,21]$. For a locally convex space $X, \operatorname{cs}(X)$ is the set of all continuous seminorms on $X$ and $\mathcal{B}(X)$ the set of all bounded absolutely convex subsets of $X$. Given locally convex spaces $X$ and $Y$, we write $L(X, Y)$ (or $L_{b}(X, Y)$ if we want to be more specific) the space of all continuous linear maps from $X$ into $Y$ endowed with the topology of uniform convergence on the bounded subsets of $X$. If $X$ and $Y$

doi:10.5186/aasfm.2012.3723

2010 Mathematics Subject Classification: Primary 47B33; Secondary 46E40, 46A04, 46A45, 46A08, 47B07, 47B38.

Key words: Composition operators, weighted spaces of analytic functions, spaces of vectorvalued analytic functions, locally convex spaces, Köthe echelon spaces.

The research of the first three authors was partially supported by MEC and FEDER Project MTM2010-15200, by GVA, Projects GV/2010/040 and Prometeo/2008/101, and Universidad Politécnica de Valencia, Project Ref. 2773. 
are Banach spaces, the topology on $L_{b}(X, Y)$ is the operator norm. A linear operator $T: X \rightarrow Y$ is called bounded if it maps a neighbourhood of $X$ into a bounded set in $Y$. Clearly every bounded operator $T$ is continuous. An operator $T: X \rightarrow Y$ is called Montel if it maps bounded sets in $X$ into relatively compact subsets of $Y$, and it is called compact (cf. [15]) if it maps a neighbourhood of $X$ into a relatively compact subset of $Y$. If $X$ and $Y$ are Banach spaces, an operator $T: X \rightarrow Y$ is Montel if and only if it is compact.

We next describe the object of our study. Let $X$ and $Y$ be complete, barrelled locally convex spaces. For an analytic self-map $\varphi$ of $\mathbf{D}$ and an analytic map $\psi: \mathbf{D} \rightarrow$ $L_{b}(X, Y)$ we consider the operator

$$
W_{\psi, \varphi}: H(\mathbf{D}, X) \longrightarrow H(\mathbf{D}, Y), \quad f \longmapsto \psi(f \circ \varphi) .
$$

This notation will be kept through the whole article. In Section 2 we show that it is well defined and continuous and we characterize when it is bounded, Montel or compact. Since $H(\mathbf{D})$ is not a Banach space, it is natural to investigate the unweighted operator $W_{\psi, \varphi}: H(\mathbf{D}, X) \rightarrow H(\mathbf{D}, Y)$ for analytic functions with values in spaces $X$ and $Y$ more general than Banach spaces. Our main result in Section 2 is Theorem 9. It requires a lemma on compact sets, Lemma 8, that uses tensor product techniques and a result due to Ruess [23]. Concrete examples in case $\psi$ is constant and in case $X$ and $Y$ are Köthe echelon spaces are given in Sections 3 and 4.

In Section 3 we turn our attention to the setting of weighted spaces of vectorvalued analytic functions, extending the work of Laitila and Tylli [16]. A weight $v: \mathbf{D} \rightarrow] 0, \infty[$ is a strictly positive, continuous, radial $(v(z)=v(|z|)$ for every $z \in \mathbf{D})$ function, that is non-increasing with respect to $|z|$. Here are the most important examples of weights:

- The standard weights are $v(z)=(1-|z|)^{\alpha}, \alpha>0$.

- $v(r)=\exp \left(-\frac{1}{(1-r)^{\alpha}}\right), \quad \alpha>0$.

- $v(r)=(1-\log (1-r))^{-\alpha}, \alpha>0$.

For a weight $v$ and a locally convex space $X$, we define

$$
H_{v}^{\infty}(\mathbf{D}, X):=\left\{f \in H(\mathbf{D}, X) ; \sup _{z \in \mathbf{D}} v(z) q(f(z))<\infty \text { for all } q \in \operatorname{cs}(X)\right\} .
$$

It is a Banach space in case $X$ is Banach, and it is denoted by $H_{v}^{\infty}(\mathbf{D})$ if $X$ is the set of complex numbers. The isomorphic classification of the spaces $H_{v}^{\infty}(\mathbf{D})$ was recently obtained by Lusky [19]. Obviously, in case that $v \equiv 1$ the space $H_{v}^{\infty}(\mathbf{D}, X)$ coincides with the space $H^{\infty}(\mathbf{D}, X)$ of bounded analytic functions $f: \mathbf{D} \rightarrow X$. When $X$ and $Y$ are Banach spaces, Laitila and Tylli characterized properties such as boundedness, compactness and weak compactness of $W_{\psi, \varphi}: H_{v}^{\infty}(\mathbf{D}, X) \rightarrow H_{w}^{\infty}(\mathbf{D}, Y)$ in terms of the weights $v$ and $w$ as well as the inducing maps. Related results for similar operators between weighted spaces of analytic functions with values in a Banach space defined by systems of weights were obtained by Manhas [20]. In Section 3 we present characterizations of continuous, bounded or Montel operators $W_{\psi, \varphi}$ in case $X$ and $Y$ are complete, barrelled spaces. Our main result is Theorem 14. Examples in Section 4 and Theorems 16 and 22 emphasize the different behaviour between the weighted and the unweighted cases and between the Banach valued and Fréchet valued functions. More precisely, we show in Theorem 22 that certain associated operator $T_{\psi}$ need not to be Montel even if $\psi(z)$ is Montel for each $z \in \mathbf{D}$, thus showing that the conclusion of Theorem 16 does not hold without the assumption $\psi \in H_{w}^{0}\left(\mathbf{D}, L_{b}(X, Y)\right)$, and that 
the equivalence in Theorem 9 fails in the weighted case. An example in the Banach valued case is given in [16, Example 4.1] for $f_{i}(z)=z^{i}, i \in \mathbf{N}, w(z)=1$ and $\lambda_{1}=\ell_{1}$. Our results are also a far reaching extension of some theorems in [6] and [8]. New techniques, and a systematic use of the Cesàro means of the Taylor polynomials, are needed in the present more general setting.

The operators investigated in this paper contain several classes of concrete linear operators. For example, in case that $X=Y=\mathbf{C}$ and $\psi$ is an analytic map $\mathbf{D} \rightarrow \mathbf{C}$ we obtain the weighted composition operator $f \mapsto \psi \cdot(f \circ \varphi)$ which combines the classical composition operator $C_{\varphi}: f \mapsto f \circ \varphi$ with the pointwise multiplication operator $M_{\psi}: f \mapsto \psi \cdot f$. For more information about composition operators, we refer the reader to the excellent monographs of Cowen and MacCluer [12] and Shapiro [24]. Many authors have studied composition operators, weighted composition operators and multiplication operators on various spaces of analytic functions. We refer, for instance, to the following articles and to the references therein: [4], [5], [6], [7], [8], [9], [10], [11], [17], [18] and [25].

\section{Operator-weighted composition operators acting between $H(\mathbf{D}, X)$ and $H(\mathbf{D}, Y)$}

The following auxiliary result is necessary in the case of analytic functions with values in barrelled locally convex spaces. It is trivial for Banach valued functions, because the duality bilinear function $\left(x, x^{\prime}\right) \rightarrow\left\langle x, x^{\prime}\right\rangle$ is continuous on $X \times X_{b}^{\prime}$ in this case.

Lemma 1. Let $X$ be a complete barrelled locally convex space. Let $h_{1}: \mathbf{D} \rightarrow X$ and $h_{2}: \mathbf{D} \rightarrow X_{b}^{\prime}$ be analytic mappings. Then

$$
h: \mathbf{D} \longrightarrow \mathbf{C}, \quad z \longmapsto h(z)=\left\langle h_{1}(z), h_{2}(z)\right\rangle
$$

belongs to $H(\mathbf{D})$.

Proof. First, for $z \neq z_{0} \in \mathbf{D}$,

$$
\begin{aligned}
\frac{h(z)-h\left(z_{0}\right)}{z-z_{0}} & =\frac{\left\langle h_{1}(z), h_{2}(z)\right\rangle-\left\langle h_{1}\left(z_{0}\right), h_{2}\left(z_{0}\right)\right\rangle}{z-z_{0}} \\
& =\left\langle\frac{h_{1}(z)-h_{1}\left(z_{0}\right)}{z-z_{0}}, h_{2}(z)\right\rangle+\left\langle h_{1}\left(z_{0}\right), \frac{h_{2}(z)-h_{2}\left(z_{0}\right)}{z-z_{0}}\right\rangle .
\end{aligned}
$$

Since $\lim _{z \rightarrow z_{0}} \frac{h_{2}(z)-h_{2}\left(z_{0}\right)}{z-z_{0}}=h_{2}^{\prime}\left(z_{0}\right)$ in $X_{b}^{\prime}$, we get

$$
\lim _{z \rightarrow z_{0}}\left\langle h_{1}\left(z_{0}\right), \frac{h_{2}(z)-h_{2}\left(z_{0}\right)}{z-z_{0}}\right\rangle=\left\langle h_{1}\left(z_{0}\right), h_{2}^{\prime}\left(z_{0}\right)\right\rangle .
$$

Now, to complete the proof, we have to show that the first summand tends to $\left\langle h_{1}^{\prime}\left(z_{0}\right), h_{2}\left(z_{0}\right)\right\rangle$. To see this, fix a sequence $\left(z_{k}\right)_{k \in \mathbf{N}} \subset \mathbf{D}$ such that $z_{k} \rightarrow z_{0}, z_{k} \neq z_{0}$. Put $x_{k}:=\frac{h_{1}\left(z_{k}\right)-h_{1}\left(z_{0}\right)}{z_{k}-z_{0}}$ for every $k \in \mathbf{N}$ and $x_{0}=h_{1}^{\prime}\left(z_{0}\right)$. Since $h_{1}: \mathbf{D} \rightarrow X$ is analytic, we know that $x_{k} \rightarrow x_{0}$ in $X$. There is $0<r_{0}<1$ such that $\overline{B\left(z_{0}, r_{0}\right)} \subset \mathbf{D}$. Find $k_{0} \in \mathbf{N}$ such that $\left|z_{k}-z_{0}\right| \leq r_{0}$ for $k \geq k_{0}$. Since $h_{2}$ is analytic, $x_{k}^{\prime}:=h_{2}\left(z_{k}\right) \rightarrow$ $h_{2}\left(z_{0}\right)=: x_{0}^{\prime}$ in $X_{b}^{\prime}$. Moreover, $\left\{h_{2}\left(z_{k}\right): k \geq k_{0}\right\} \cup\left\{h_{2}\left(z_{0}\right)\right\} \subset h_{2}\left(\overline{B\left(z_{0}, r_{0}\right)}\right)=: K \subset X^{\prime}$ is compact in $X_{b}^{\prime}$ by the continuity of $h_{2}$. Given $\varepsilon>0$, we apply that $X$ is barrelled to get that $K$ is equicontinuous in $X^{\prime}$. Hence there is $k_{1} \geq k_{0}$ such that for every $k \geq k_{1}$, 


$$
\left|\left\langle x_{0}, x_{k}^{\prime}-x_{0}^{\prime}\right\rangle\right|<\frac{\varepsilon}{2} \text { and } \sup _{u \in K}\left|u\left(x_{k}-x_{0}\right)\right|<\frac{\varepsilon}{2} .
$$

Hence

$$
\begin{aligned}
\left|\left\langle x_{k}, x_{k}^{\prime}\right\rangle-\left\langle x_{0}, x_{0}^{\prime}\right\rangle\right| & =\left|\left\langle x_{k}-x_{0}+x_{0}, x_{k}^{\prime}\right\rangle-\left\langle x_{0}, x_{0}^{\prime}\right\rangle\right| \leq\left|\left\langle x_{k}-x_{0}, x_{k}^{\prime}\right\rangle\right|+\left|\left\langle x_{0}, x_{k}^{\prime}-x_{0}^{\prime}\right\rangle\right| \\
& \leq \sup _{u \in K}\left|u\left(x_{k}-x_{0}\right)\right|+\left|\left\langle x_{0}, x_{k}^{\prime}-x_{0}^{\prime}\right\rangle\right|<\varepsilon
\end{aligned}
$$

for every $k \geq k_{1}$. The proof is complete.

Proposition 2. Let $X$ and $Y$ be complete barrelled locally convex spaces. Let $\varphi: \mathbf{D} \rightarrow \mathbf{D}$ and $\psi: \mathbf{D} \rightarrow L_{b}(X, Y)$ be analytic mappings. Then the operator

$$
W_{\psi, \varphi}: H(\mathbf{D}, X) \longrightarrow H(\mathbf{D}, Y), \quad f \longmapsto\left(W_{\psi, \varphi} f\right)(z)=\psi(z)[f(\varphi(z))]
$$

is well-defined.

Proof. We have to show that $W_{\psi, \varphi} f \in H(\mathbf{D}, Y)$ for every $f \in H(\mathbf{D}, X)$. Since $Y$ is complete, by a classical result of Grothendieck [14, Section 16.7], it is enough to prove that $y^{\prime} \circ W_{\psi, \varphi} f \in H(\mathbf{D})$ for every $y^{\prime} \in Y^{\prime}$. Fix $y^{\prime} \in Y^{\prime}$ and consider $T_{y^{\prime}}: L_{b}(X, Y) \rightarrow X_{b}^{\prime}, A \rightarrow y^{\prime} \circ A$. Then $T_{y^{\prime}}$ is linear and continuous. Since $\psi \in$ $H\left(\mathbf{D}, L_{b}(X, Y)\right)$ we have $T_{y^{\prime}} \circ \psi \in H\left(\mathbf{D}, X_{b}^{\prime}\right)$. Moreover, $f \circ \varphi \in H(\mathbf{D}, X)$ if $f \in$ $H(\mathbf{D}, X)$. We apply Lemma 1 to obtain that the map from $\mathbf{D}$ to $\mathbf{C}$ given by

$$
z \longmapsto y^{\prime}(\psi(z)[(f \circ \varphi)(z)])=\left\langle\left(y^{\prime} \circ \psi\right)(z),(f \circ \varphi)(z)\right\rangle
$$

is analytic.

The following auxiliary linear operator plays an important role in our characterizations. It was introduced in the case of Banach valued functions by Laitila and Tylli in Section 3 of [16].

Definition 3. Let $\psi: \mathbf{D} \rightarrow L_{b}(X, Y)$ be analytic. We consider the operator

$$
T_{\psi}: X \longrightarrow H(\mathbf{D}, Y), \quad x \longmapsto T_{\psi}(x): \mathbf{D} \rightarrow Y, T_{\psi}(x)(z)=\psi(z)[x] .
$$

For $x \in X$ the operator $R_{x}: L_{b}(X, Y) \longrightarrow Y, A \mapsto A x$ is linear and continuous and satisfies $T_{\psi}(x)=R_{x} \circ \psi$. Thus, $T_{\psi}(x)$ is analytic as a composition of a linear continuous operator with an analytic map. Moreover, $T_{\psi}$ is linear by the definition of $R_{x}$.

Remark 4. (i) The auxiliary operator $T_{\psi}$ inherits the ideal properties of $W_{\psi, \varphi}$. Indeed, the operator $A: X \rightarrow H(\mathbf{D}, X),(A x)(z)=f_{x}(z)=x$ is linear and continuous and $T_{\psi}=W_{\psi, \varphi} \circ A$, from where the conclusion follows.

(ii) All the operators $\psi(z): X \rightarrow Y, z \in \mathbf{D}$ inherit the properties of $T_{\psi}$. To see this, consider the evaluation operator $\delta_{z}: H(\mathbf{D}, Y) \rightarrow Y, f \rightarrow f(z)$. Since $\delta_{z}$ is continuous and $\delta_{z}\left(T_{\psi}(x)\right)=T_{\psi}(x)(z)=\psi(z)[x]$ for every $x \in X$ and every $z \in \mathbf{D}$, the conclusion follows.

Proposition 5. Let $X$ and $Y$ be complete barrelled locally convex spaces. Let $\varphi: \mathbf{D} \rightarrow \mathbf{D}$ and $\psi: \mathbf{D} \rightarrow L_{b}(X, Y)$ be analytic mappings. Then the operator $W_{\psi, \varphi}: H(\mathbf{D}, X) \rightarrow H(\mathbf{D}, Y)$ is continuous.

Proof. Fix a compact subset $K$ of $\mathbf{D}$ as well as a seminorm $q \in c s(Y)$. Since $\psi: \mathbf{D} \rightarrow L_{b}(X, Y)$ is analytic and $X$ is barrelled, the set $\psi(K)$ is relatively compact 
in $L_{b}(X, Y)$, hence equicontinuous. Find a seminorm $p \in \operatorname{cs}(X)$ such that

$$
q(\psi(z)[x]) \leq p(x) \text { for every } z \in K \text { and every } x \in X \text {. }
$$

Finally,

$$
\sup _{z \in K} q(\psi(z)[f(\varphi(z))]) \leq \sup _{z \in K} p(f(\varphi(z))) \leq \sup _{\xi \in \varphi(K)} p(f(\xi)) .
$$

This shows that the operator is continuous.

Theorem 6. Let $X$ and $Y$ be complete barrelled locally convex spaces. Let $\varphi: \mathbf{D} \rightarrow \mathbf{D}$ and $\psi: \mathbf{D} \rightarrow L_{b}(X, Y), \psi \neq 0$, be analytic mappings. Then the operator $W_{\psi, \varphi}: H(\mathbf{D}, X) \rightarrow H(\mathbf{D}, Y)$ is bounded if and only if the following conditions hold:

(a) $T_{\psi}: X \rightarrow H(\mathbf{D}, Y)$ is bounded.

(b) There is $0<r_{0}<1$ such that $|\varphi(z)| \leq r_{0}$ for every $z \in \mathbf{D}$.

Proof. First we assume that conditions (a) and (b) hold. From (a) it follows that there is a seminorm $p \in c s(X)$ such that for every compact set $K \subset \mathbf{D}$ and for every $q \in \operatorname{cs}(Y)$ we can find $\lambda>0$ such that

$$
\sup _{z \in K} q\left(T_{\psi}(x)(z)\right)=\sup _{z \in K} q(\psi(z)[x]) \leq \lambda p(x) \text { for every } x \in X .
$$

Select $K_{0}:=\left\{z \in \mathbf{C} ;|z| \leq r_{0}\right\}$ with $r_{0}$ as in condition (b) and define

$$
P(f):=\sup _{\xi \in K_{0}} p(f(\xi)) \in \operatorname{cs}(H(\mathbf{D}, X)) .
$$

For every compact set $K \subset \mathbf{D}$ and $q \in \operatorname{cs}(Y)$ we put

$$
Q_{K, q}\left(W_{\psi, \varphi} f\right):=\sup _{z \in K} q(\psi(z)[f(\varphi(z))])
$$

and obtain, from (b),

$$
Q_{K, q}\left(W_{\psi, \varphi} f\right) \leq \lambda \sup _{z \in K} p(f(\varphi(z))) \leq \lambda \sup _{\xi \in K_{0}} p(f(\xi)) .
$$

This shows that $W_{\psi, \varphi}: H(\mathbf{D}, X) \rightarrow H(\mathbf{D}, Y)$ is bounded.

It remains to show the converse. Obviously $T_{\psi}$ is bounded by Remark 4 . Since $W_{\psi, \varphi}: H(\mathbf{D}, X) \rightarrow H(\mathbf{D}, Y)$ is bounded, we find $0<r_{0}<1$ and $p \in c s(X)$ such that for every $0<r<1$ and every $q \in \operatorname{cs}(Y)$ there is $\lambda>0$ such that for every $f \in H(\mathbf{D}, X)$ we have

$$
\sup _{|z| \leq r} q(\psi(z)[f(\varphi(z))]) \leq \lambda \sup _{|\xi| \leq r_{0}} p(f(\xi)) .
$$

Applying this inequality for the maps $f_{k}: \mathbf{D} \rightarrow X$ given by $f_{k}(z)=z^{k} x, k \in \mathbf{N}_{0}$, which clearly belong to the space $H(\mathbf{D}, X)$ for each $x \in X$, we obtain

$$
\sup _{|z| \leq r}|\varphi(z)|^{k} q(\psi(z)[x])=\sup _{|z| \leq r} q\left(\psi(z)\left[\varphi(z)^{k} x\right]\right) \leq \lambda \sup _{|z| \leq r_{0}} p\left(z^{k} x\right)=\lambda r_{0}^{k} p(x)
$$

for every $q \in \operatorname{cs}(Y), k \in \mathbf{N}_{0}$ and $x \in X$. In particular, for $k=0$ and $0<r<1$ arbitrary, we get

$$
\sup _{|z| \leq r} q(\psi(z)[x]) \leq \lambda p(x)
$$

for every $q \in \operatorname{cs}(Y)$, which implies $\psi(z)[x]=0$ for every $z \in \mathbf{D}$ if $p(x)=0$. Now, since $\psi \neq 0$ by assumption, there are $z_{0} \in \mathbf{D}, x_{0} \in X$ such that $\psi\left(z_{0}\right)\left[x_{0}\right] \neq 0$. In particular, $p\left(x_{0}\right)>0$. Find $q_{0} \in \operatorname{cs}(Y)$ with $q_{0}\left(\psi\left(z_{0}\right)\left[x_{0}\right]\right)>0$. 
Next, we apply (2.1) to get, for each $0<r<1$,

$$
0<q_{0}\left(\psi\left(z_{0}\right)\left[x_{0}\right]\right) \sup _{|z| \leq r}|\varphi(z)|^{k} \leq \lambda r_{0}^{k} p\left(x_{0}\right),
$$

for every $k \in \mathbf{N}_{0}$. Hence

$$
\sup _{|z| \leq r}|\varphi(z)|^{k} \leq \frac{\lambda r_{0}^{k} p\left(x_{0}\right)}{q_{0}\left(\psi\left(z_{0}\right)\left[x_{0}\right]\right)},
$$

for every $k \in \mathbf{N}_{0}$. Taking the $k$-th roots and letting $r$ go to 1 we obtain $|\varphi(z)| \leq r_{0}$ for every $z \in \mathbf{D}$, and (b) follows.

In our next results, we use the following notation (cf. [15]): $H(\mathbf{D})_{c o}^{\prime}$ is the dual of $H(\mathbf{D})$ endowed with the topology of uniform convergence on the convex compact sets, and $L_{e}\left(H(\mathbf{D})_{c o}^{\prime}, Y\right)$ is the space of all linear and continuous operators endowed with the topology of uniform convergence on the equicontinuous subsets of $H(\mathbf{D})^{\prime}$. The space $L_{e}\left(H(\mathbf{D})_{c o}^{\prime}, Y\right)$ coincides with the $\varepsilon$ product of Schwartz $H(\mathbf{D}) \varepsilon Y$ if $Y$ is complete; see [15, page 242]. In what follows, if $\langle E, F\rangle$ is a dual pair and $A \subset E$, $A^{\circ}:=\{u \in F||\langle x, u\rangle \mid \leq 1$ for each $x \in A\}$ is the absolute polar of $A$ in $F$. By the bipolar theorem, the bipolar $A^{\circ 0}$ of a subset $A$ of $E$ is the closure in the weak topology $\sigma(E, F)$ of the absolutely convex hull $\Gamma(A)$ of the set $A$.

Lemma 7. Let $Y$ be a complete locally convex space. A bounded subset $H \subset$ $L_{e}\left(H(\mathbf{D})_{c o}^{\prime}, Y\right)$ is precompact if and only if $H\left(U^{\circ}\right)$ is precompact in $Y$ for every $U \in \mathcal{U}_{0}(H(\mathbf{D}))$.

Proof. By [23, Theorem 1.5], a subset $H \subset L_{e}\left(H(\mathbf{D})_{c o}^{\prime}, Y\right)$ is precompact if and only if (a) $H\left(U^{\circ}\right)$ is precompact in $Y$ for every absolutely convex neighbourhood $U$ in $H(\mathbf{D})$ and $(\mathrm{b}) H^{\prime}\left(y^{\prime}\right):=\left\{y^{\prime} \circ h ; h \in H\right\}$ is precompact in $H(\mathbf{D})$ for every $y^{\prime} \in Y^{\prime}$. Since $H(\mathbf{D}, Y) \cong L_{e}\left(H(\mathbf{D})_{c o}^{\prime}, Y\right)$ by $[14$, Section 16.7], every bounded subset $H$ of $L_{e}\left(H(\mathbf{D})_{c o}^{\prime}, Y\right)$ satisfies (b) by Montel's theorem.

Lemma 8. Let $Y$ be a complete locally convex space and let $C$ be a bounded subset of $H(\mathbf{D}, Y)$. The set $C$ is precompact if and only if for every compact subset $K$ of $\mathbf{D}$ the set $C(K):=\{f(z) ; z \in K, f \in C\}$ is precompact in $Y$.

Proof. To present the proof we must briefly recall the isomorphism $H(\mathbf{D}, Y) \cong$ $L_{e}\left(H(\mathbf{D})_{c o}^{\prime}, Y\right)\left(\left[14\right.\right.$, Section 16.7]). If $T \in L_{e}\left(H(\mathbf{D})_{c o}^{\prime}, Y\right)$, the corresponding element in $H(\mathbf{D}, Y)$ is defined by $f_{T}(z):=T\left(\delta_{z}\right)$, where $\delta_{z} \in H(\mathbf{D})^{\prime}$ is the evaluation at the point $z \in \mathbf{D}$. Conversely if $g \in H(\mathbf{D}, Y)$, the operator $T_{g} \in L_{e}\left(H(\mathbf{D})_{c o}^{\prime}, Y\right)$ is the unique continuous extension of the linear map defined as $T_{g}\left(\delta_{z}\right):=g(z)$. We use this identification in the rest of the proof.

First assume that $C$ is a precompact subset of $H(\mathbf{D}, Y)$. Fix a compact subset $K$ of $\mathbf{D}$. We prove that $C(K)$ is precompact in $Y$. Set

$$
U:=\left\{f \in H(\mathbf{D}) ; \sup _{z \in K}|f(z)| \leq 1\right\}=\left\{\delta_{z} ; z \in K\right\}^{\circ},
$$

the polar of $\left\{\delta_{z} ; z \in K\right\} \subset H(\mathbf{D})^{\prime}$ taken in $H(\mathbf{D})$. Hence $U^{\circ}=\left\{\delta_{z} ; z \in K\right\}^{\circ \circ}=$ $\overline{\Gamma\left\{\delta_{z} ; z \in K\right\}}$. Since $C$ is precompact in $H(\mathbf{D}, Y)$, we apply Lemma 7 to conclude that $C\left(U^{\circ}\right)$ is precompact in $Y$. But

$$
C\left(\left\{\delta_{z} ; z \in K\right\}\right)=\left\{f\left(\delta_{z}\right)=f(z) ; f \in C, z \in K\right\} \subset C\left(U^{\circ}\right)
$$

and $C(K)$ is precompact, too. 
To prove the converse we apply Lemma 7 . Fix an absolutely convex neighbourhood $U$ in $H(\mathbf{D})$ and select $\varepsilon>0$ and a compact subset $K$ of $\mathbf{D}$ such that $\varepsilon U^{\circ} \subset$ $\overline{\Gamma\left\{\delta_{z} ; z \in K\right\}}$, the closure taken in the topology $\sigma\left(H(\mathbf{D})^{\prime}, H(\mathbf{D})\right)$. We have to show that $C\left(U^{\circ}\right)$ is precompact in $Y$. By assumption, the set $D:=\overline{\Gamma\{f(z) ; z \in K, f \in C\}}$ is precompact in $Y$. Next, $\varepsilon C\left(U^{\circ}\right)=C\left(\varepsilon U^{\circ}\right)=\bigcup_{h \in C} h\left(\varepsilon U^{\circ}\right)$. Moreover, $h\left(\varepsilon U^{\circ}\right) \subset$ $h\left(\overline{\Gamma\left\{\delta_{z} ; z \in K\right\}}\right)$ and $h: H(\mathbf{D})_{c o}^{\prime} \rightarrow Y$ continuous. Thus

$$
h\left(\overline{\Gamma\left\{\delta_{z} ; z \in K\right\}}\right) \subset \overline{h\left(\Gamma\left(\left\{\delta_{z} ; z \in K\right\}\right)\right.} \subset \overline{\Gamma h\left(\left\{\delta_{z} ; z \in K\right\}\right)} \subset D
$$

since $h \in C$. Therefore,

$$
\bigcup_{h \in C} h\left(\varepsilon U^{\circ}\right) \subset D
$$

and $C\left(U^{\circ}\right)$ is precompact in $Y$.

Theorem 9. Let $X$ and $Y$ be complete barrelled locally convex spaces. Let $\varphi: \mathbf{D} \rightarrow \mathbf{D}$ and $\psi: \mathbf{D} \rightarrow L_{b}(X, Y)$ be analytic maps. The following assertions are equivalent:

(a) $W_{\psi, \varphi}: H(\mathbf{D}, X) \rightarrow H(\mathbf{D}, Y)$ is Montel.

(b) $T_{\psi}: X \rightarrow H(\mathbf{D}, Y)$ is Montel.

(c) $\psi(z): X \rightarrow Y$ is Montel for all $z \in \mathbf{D}$.

Proof. Remark 4 implies that $(c)$ follows from $(a)$.

To prove that $(c)$ implies $(b)$, assume that $\psi(z): X \rightarrow Y$ is a Montel operator for every $z \in \mathbf{D}$. In order to prove that $T_{\psi}$ is a Montel operator, we approximate it by a sequence of Montel operators in $L_{b}(X, H(\mathbf{D}, Y))$. The function $\psi$ is analytic with values in $L_{b}(X, Y)$, and then

$$
\psi(z)=\sum_{m=0}^{\infty} A_{m} z^{m},
$$

where $A_{m} \in L_{b}(X, Y)$ and the series converges in $L_{b}(X, Y)$ uniformly on the compact sets of D. First, we check that the coefficients $A_{m}$ are Montel operators from $X$ to $Y$. Indeed, $A_{0}=\psi(0)$ which is a Montel operator. The rest of the coefficients are Montel operators by the Cauchy Integral Formula for derivatives.

Now, we consider the Taylor polynomials of $\psi, \psi_{n}(z)=\sum_{m=0}^{n} A_{m} z^{m}, n=$ $0,1,2, \ldots$, and the corresponding auxiliary operators $T_{\psi_{n}}$. We will see that $T_{\psi_{n}}$ is a Montel operator for each $n$, and that $T_{\psi_{n}}$ tends to $T_{\psi}$ in $L_{b}(X, H(\mathbf{D}, Y))$ when $n$ tends to infinity. Let $B$ be a bounded set in $X$, then $A_{m}(B)$ is relatively compact in $Y$ for each $m=0,1, \ldots, n$. The closure of the absolutely convex hull of the union of these sets is compact in $Y$, that is, the set

$$
K:=\overline{\Gamma\left(\bigcup_{m=0}^{n} A_{m}(B)\right)}
$$

is compact in $Y$. Consequently, if $0<R_{0}<1$, we obtain

$$
\left\{\sum_{m=0}^{n} A_{m}(x) z^{m}:|z| \leq R_{0}, x \in B\right\} \subset n \cdot K,
$$

which proves that $T_{\psi_{n}}$ is a Montel operator. To conclude the proof, we see that $T_{\psi_{n}}$ tends to $T_{\psi}$ in $L_{b}(X, H(\mathbf{D}, Y))$. If $B$ is a bounded set in $X, 0<R<1$ and $q$ is a 
continuous seminorm in $Y$, we have

$$
\sup _{x \in B} \sup _{|z| \leq R} q\left(\left(T_{\psi}-T_{\psi_{n}}\right)(x)[z]\right)=\sup _{x \in B} \sup _{|z| \leq R} q\left(\sum_{m=n+1}^{\infty} A_{m}(x) z^{m}\right),
$$

that tends to 0 when $n$ tends to infinity, since $\psi(z)=\sum_{m=0}^{\infty} A_{m} z^{m}$ in $L_{b}(X, Y)$ uniformly on compact sets.

It remains to show that (b) implies (a). Take a bounded set $B$ in $H(\mathbf{D}, X)$. Since $B$ is bounded for every compact set $K$ in $\mathbf{D}$ and every continuous seminorm $p$ in $X$, we have

$$
\sup _{z \in K} \sup _{f \in B} p(f(z))<\infty
$$

In particular, as $\varphi(K)$ is a compact set in $\mathbf{D}$, we also have

$$
\sup _{z \in K} \sup _{f \in B} p(f(\varphi(z)))<\infty
$$

for every continuous seminorm $p$ in $X$. Therefore, the set

$$
B_{K}:=\{f(\varphi(z)): z \in K, f \in B\}
$$

is bounded in $X$, for every compact set $K$ in $\mathbf{D}$. By hypothesis, $T_{\psi}\left(B_{K}\right)$ is precompact in $H(\mathbf{D}, Y)$. By Lemma 8 , for every compact set $L$ in $\mathbf{D}$, the set

$$
\{\psi(\xi)[f(\varphi(z))]: \xi \in L, z \in K, f \in B\}
$$

is a precompact set in $Y$. Therefore, the set $\{\psi(z)[f(\varphi(z))]: z \in K, f \in B\}$ is precompact in $Y$, for each compact set $K$ in D. Since $W_{\psi, \varphi}(f)[z]=\psi(z)[f(\varphi(z))]$ for $f \in B$ and $z \in K$, by Lemma 8 again, we conclude that $W_{\psi, \varphi}(B)$ is precompact in $H(\mathbf{D}, Y)$. Then, $(b)$ implies $(a)$.

Proposition 10. Let $X, Y$ be complete barrelled locally convex spaces. Let $\varphi: \mathbf{D} \rightarrow \mathbf{D}$ and $\psi: \mathbf{D} \rightarrow L_{b}(X, Y)$ be analytic maps. Then the following assertions are equivalent:

(1) The operator $W_{\psi, \varphi}: H(\mathbf{D}, X) \rightarrow H(\mathbf{D}, Y)$ is compact.

(2) The following conditions hold:

(a) $T_{\psi}: X \rightarrow H(\mathbf{D}, Y)$ is compact.

(b) There is $0<r_{0}<1$ such that $|\varphi(z)| \leq r_{0}$ for every $z \in \mathbf{D}$.

Proof. First, we suppose that $W_{\psi, \varphi}$ is compact. Since $T_{\psi}$ inherits the properties of $W_{\psi, \varphi}$ by Remark 4 (i), we have that $T_{\psi}$ is compact. Moreover, the condition (b) follows from Theorem 6 , since the operator $W_{\psi, \varphi}$ is bounded.

To prove that (2) implies (1), we select $r_{0}$ as in condition 2.(b) and set $K_{0}=$ $\left\{\rho:|\rho| \leq r_{0}\right\}$. From 2.(a) there exists a continuous seminorm $p$ on $X$ such that $T_{\psi}\left(U_{p}\right)$ is precompact in $H(\mathbf{D}, Y)$ where $U_{p}:=\{x \in X: p(x) \leq 1\}$. Then

$$
\{\psi(z) x: z \in K, p(x) \leq 1\}
$$

is precompact in $Y$, for each compact set $K$ in $\mathbf{D}$, by Lemma 8. We consider

$$
V:=\left\{g \in H(\mathbf{D}, X): \sup _{\rho \in K_{0}} p(g(\rho)) \leq 1\right\} \in \mathcal{U}_{0}(H(\mathbf{D}, X)) .
$$

We show that $W_{\psi, \varphi}(V)$ is precompact in $H(\mathbf{D}, Y)$. From Lemma 8 it suffices to check that for all compact sets $K$ in $\mathbf{D}$, the sets

$$
\left\{W_{\psi, \varphi}(g)(z): z \in K, g \in V\right\}
$$


are precompact in $Y$.

Let $K$ be a compact subset of $\mathbf{D}$. If $z \in K$ and $g \in V$, since $\varphi(z) \in K_{0}$, we also have $p(g(\varphi(z))) \leq 1$. Then from $(2.2)$ we have that

$$
\left\{W_{\psi, \varphi}(g)(z): z \in K, g \in V\right\}=\{\psi(z)(g(\varphi(z))): z \in K, g \in V\}
$$

is precompact in $Y$.

\section{Operator-weighted composition operators acting between weighted spaces $H_{v}^{\infty}(\mathbf{D}, X)$ and $H_{w}^{\infty}(\mathbf{D}, Y)$}

In this section we study the operator-weighted composition operators acting between weighted spaces. In the formulation of our results we need the associated weights of [2]. For a weight $v$ on $\mathbf{D}$ the corresponding associated weight $\tilde{v}$ is defined by

$$
\tilde{v}(z):=\frac{1}{\sup \left\{|f(z)| ; f \in H_{v}^{\infty}(\mathbf{D}),\|f\|_{v} \leq 1\right\}}=\frac{1}{\left\|\delta_{z}\right\|_{H_{v}^{\infty}(\mathbf{D})^{\prime}}}, \quad z \in \mathbf{D}
$$

where $\|f\|_{v}:=\sup _{z \in \mathbf{D}} v(z)|f(z)|$ and $\delta_{z}$ denotes the point evaluation of $z \in \mathbf{D}$. By [2] we know that associated weights are continuous, $\tilde{v} \geq v>0$ and that for each $z \in \mathbf{D}$ we can find $f_{z} \in H_{v}^{\infty}(\mathbf{D}),\|f\|_{v} \leq 1$, such that $\left|f_{z}(z)\right|=\frac{1}{\tilde{v}(z)}$. It is well known that $H_{\tilde{v}}^{\infty}(\mathbf{D})$ is isometrically isomorphic to $H_{v}^{\infty}(\mathbf{D})$. Applying the Hahn-Banach theorem we conclude

Lemma 11. Let $X$ be a complete barrelled locally convex space. For every $p \in \operatorname{cs}(X)$ and every $f \in H_{v}^{\infty}(\mathbf{D}, X)$ we have

$$
\sup _{\xi \in \mathbf{D}} \tilde{v}(\xi) p(f(\xi))=\sup _{z \in \mathbf{D}} v(z) p(f(z)) .
$$

Proposition 12. Let $X$ and $Y$ be complete barrelled locally convex spaces. Let $\varphi: \mathbf{D} \rightarrow \mathbf{D}$ and $\psi: \mathbf{D} \rightarrow L_{b}(X, Y)$ be analytic maps. The operator $W_{\psi, \varphi}: H_{v}^{\infty}(\mathbf{D}, X)$ $\rightarrow H_{w}^{\infty}(\mathbf{D}, Y), f \rightarrow\left(W_{\psi, \varphi} f\right)(z)=\psi(z)[f(\varphi(z))]$ is continuous if and only if the set

$$
\left\{\frac{w(z)}{\tilde{v}(\varphi(z))} \psi(z) ; z \in \mathbf{D}\right\}
$$

is equicontinuous on $L_{b}(X, Y)$.

Proof. First, assume that the set $\left\{\frac{w(z)}{\tilde{v}(\varphi(z))} \psi(z) ; z \in \mathbf{D}\right\}$ is equicontinuous on $L_{b}(X, Y)$. For each $q \in \operatorname{cs}(Y)$ we can find $p \in c s(X)$ such that

$$
\frac{w(z)}{\tilde{v}(\varphi(z))} q(\psi(z)[x]) \leq p(x) \text { for every } x \in X \text { and every } z \in \mathbf{D} .
$$

Thus, for $z \in \mathbf{D}$ and $f \in H_{v}^{\infty}(\mathbf{D}, X)$, we get

$$
\begin{aligned}
w(z) q(\psi(z)[f(\varphi(z))]) & =\tilde{v}(\varphi(z)) \frac{w(z)}{\tilde{v}(\varphi(z))} q(\psi(z)[f(\varphi(z))]) \\
& \stackrel{(3.1)}{\leq} \tilde{v}(\varphi(z)) p\left(f(\varphi(z)) \leq \sup _{\xi \in \mathbf{D}} \tilde{v}(\xi) p(f(\xi))\right.
\end{aligned}
$$

and the operator $W_{\psi, \varphi}: H_{v}^{\infty}(\mathbf{D}, X) \rightarrow H_{w}^{\infty}(\mathbf{D}, Y)$ is continuous.

We prove the converse by contradiction: Assume that the set $\left\{\frac{w(z)}{\tilde{v}(\varphi(z))} \psi(z) ; z \in \mathbf{D}\right\}$ is not equicontinuous on $L_{b}(X, Y)$. Since $X$ is barrelled, this means that we can find 
an element $x \in X$ such that the set $\left\{\frac{w(z)}{\tilde{v}(\varphi(z))} \psi(z)[x] ; z \in \mathbf{D}\right\}$ is not bounded in $Y$. Thus there exists $y^{\prime} \in Y^{\prime}$ such that $\left\{\frac{w(z)}{\tilde{v}(\varphi(z))} y^{\prime}(\psi(z)[x]) ; z \in \mathbf{D}\right\}$ is not bounded in $\mathbf{C}$, and we can find a sequence $\left(z_{n}\right)_{n} \subset \mathbf{D}$ such that

$$
\frac{w\left(z_{n}\right)}{\tilde{v}\left(\varphi\left(z_{n}\right)\right)}\left|y^{\prime}\left(\psi\left(z_{n}\right)[x]\right)\right| \geq n
$$

for every $n \in \mathbf{N}$. We select $f_{n} \in H(\mathbf{D})$ such that $\left|f_{n}\right| \leq \frac{1}{v}, f_{n}\left(\varphi\left(z_{n}\right)\right)=1 / \tilde{v}\left(\varphi\left(z_{n}\right)\right)$, and define $g_{n}: \mathbf{D} \rightarrow X$ by

$$
g_{n}(z):=f_{n}(z) x
$$

for every $n \in \mathbf{N}$, with $x$ chosen above. The sequence $\left(g_{n}\right)_{n}$ is bounded in $H_{v}^{\infty}(\mathbf{D}, X)$ since for every $p \in \operatorname{cs}(X)$ and every $z \in \mathbf{D}$ we have

$$
v(z) p\left(g_{n}(z)\right)=v(z)\left|f_{n}(z)\right| p(x) \leq p(x) .
$$

By assumption $\left(W_{\psi, \varphi} g_{n}\right)_{n}$ is a bounded sequence in $H_{w}^{\infty}(\mathbf{D}, Y)$ and

$$
W_{\psi, \varphi}\left(g_{n}\right)(z)=\psi(z)\left[g_{n}(\varphi(z))\right]=f_{n}(\varphi(z)) \psi(z)[x] .
$$

With $y^{\prime} \in Y^{\prime}$ selected as above, we put $q(s):=\left|y^{\prime}(s)\right| \in c s(Y)$. Then there exists a constant $M>0$ such that for every $z \in \mathbf{D}$ and every $n \in \mathbf{N}$

$$
w(z) q\left(W_{\psi, \varphi} g_{n}(z)\right) \leq M
$$

which implies $n \leq \frac{w\left(z_{n}\right)}{\tilde{v}\left(\varphi\left(z_{n}\right)\right)}\left|y^{\prime}\left(\psi\left(z_{n}\right)[x]\right)\right| \leq M$ for every $n \in \mathbf{N}$. This is a contradiction.

Recall that a subset $C$ of $L(X, Y)$ is called equibounded if there is a neighbourhood $U$ in $X$ such that $\{T(x) \mid x \in U, T \in C\}$ is bounded in $Y$.

Proposition 13. Let $X$ and $Y$ be complete barrelled locally convex spaces. Moreover, let $\varphi: \mathbf{D} \rightarrow \mathbf{D}$ and $\psi: \mathbf{D} \rightarrow L_{b}(X, Y)$ be analytic maps. Then the operator $W_{\psi, \varphi}: H_{v}^{\infty}(\mathbf{D}, X) \rightarrow H_{w}^{\infty}(\mathbf{D}, Y)$ is bounded if and only if the set

$$
\left\{\frac{w(z)}{\tilde{v}(\varphi(z))} \psi(z) ; z \in \mathbf{D}\right\}
$$

is equibounded in $L_{b}(X, Y)$.

Proof. It is very similar to the proof of Proposition 12, once you have in mind that $\left\{\frac{w(z)}{\tilde{v}(\varphi(z))} \psi(z) ; z \in \mathbf{D}\right\}$ is equibounded in $L_{b}(X, Y)$ if and only if there is $p \in \operatorname{cs}(X)$ such that for every $q \in \operatorname{cs}(Y)$ there is a constant $\lambda_{q}>0$ with

$$
q\left(\frac{w(z)}{\tilde{v}(\varphi(z))} \psi(z)[x]\right) \leq \lambda_{q} p(x)
$$

for every $x \in X$ and every $z \in \mathbf{D}$.

Let $X$ be a complete barrelled locally convex space. In the proof of Theorem 14 we denote, for every $k \in \mathbf{N}_{0}$,

$$
q_{k}: H(\mathbf{D}, X) \longrightarrow X, \quad f \longmapsto \frac{f^{(k)}(0)}{k !}
$$

which is continuous, by the Cauchy Integral Formula for derivatives. 
Theorem 14. Let $X$ and $Y$ be complete barrelled locally convex spaces. Moreover, let $\varphi: \mathbf{D} \rightarrow \mathbf{D}$ and $\psi: \mathbf{D} \rightarrow L_{b}(X, Y)$ be analytic maps such that the operator $W_{\psi, \varphi}: H_{v}^{\infty}(\mathbf{D}, X) \rightarrow H_{w}^{\infty}(\mathbf{D}, Y)$ is continuous. The weighted composition operator $W_{\psi, \varphi}$ is Montel if, and only if, the following conditions are satisfied:

(a) $T_{\psi}: X \rightarrow H_{w}^{\infty}(\mathbf{D}, Y)$ is Montel.

(b) For every $B \in \mathcal{B}(X), q \in \operatorname{cs}(Y)$ and $\varepsilon>0$ there is $\left.r_{0} \in\right] 0,1[$ such that if $|\varphi(z)|>r_{0}$ and $x \in B$, then we have the following inequality

$$
\frac{w(z)}{\tilde{v}(\varphi(z))} q(\psi(z)[x]) \leq \varepsilon .
$$

Proof. Let us first assume that conditions (a) and (b) are satisfied. We will approximate the operator $W_{\psi, \varphi}$ by a sequence of Montel operators in $L_{b}\left(H_{v}^{\infty}(\mathbf{D}, X)\right.$, $\left.H_{w}^{\infty}(\mathbf{D}, Y)\right)$. To do this we consider the Cesàro sums given by

$$
C_{m}: H_{v}^{\infty}(\mathbf{D}, X) \longrightarrow H_{v}^{\infty}(\mathbf{D}, X), \quad f \longmapsto \frac{1}{m+1} \sum_{k=0}^{m} P_{k} f
$$

where $P_{k} f(z)=\sum_{\ell=0}^{k} \frac{f^{(\ell)}(0)}{\ell !} z^{\ell}$ is the $k$-th Taylor polynomial of $f$. First we want to show that $W_{\psi, \varphi} C_{m}$ is a Montel operator acting from $H_{v}^{\infty}(\mathbf{D}, X)$ to $H_{w}^{\infty}(\mathbf{D}, Y)$. The definition gives

$$
\begin{aligned}
\left(W_{\psi, \varphi} C_{m} f\right)(z) & =\psi(z)\left[C_{m} f(\varphi(z))\right]=\psi(z)\left[\frac{1}{m+1} \sum_{k=0}^{m}\left(\sum_{\ell=0}^{k} \frac{f^{(\ell)}(0)}{\ell !} \varphi(z)^{\ell}\right)\right] \\
& =\frac{1}{m+1} \sum_{k=0}^{m}\left(\sum_{\ell=0}^{k} \varphi(z)^{\ell} \psi(z) \frac{f^{(\ell)}(0)}{\ell !}\right)=\frac{1}{m+1} \sum_{k=0}^{m} \sum_{\ell=0}^{k} \varphi(z)^{\ell} T_{\psi} q_{\ell}(f)(z) .
\end{aligned}
$$

Since $T_{\psi}$ is a Montel operator and the $q_{\ell}$ are continuous we obtain that $W_{\psi, \varphi} C_{m}$ is a Montel operator for every $m \in \mathbf{N}_{0}$, as desired. Now, we show that

$$
W_{\psi, \varphi}=\lim _{m \rightarrow \infty} W_{\psi, \varphi} C_{m}
$$

in $L_{b}\left(H_{v}^{\infty}(\mathbf{D}, X), H_{w}^{\infty}(\mathbf{D}, Y)\right)$. To do this, we fix a bounded set $C$ in $H_{v}^{\infty}(\mathbf{D}, X)$ as well as $q \in \operatorname{cs}(Y)$ and have to show

$$
\sup _{f \in C} \sup _{z \in \mathbf{D}} w(z) q\left[W_{\psi, \varphi} f(z)-W_{\psi, \varphi} C_{m} f(z)\right] \rightarrow 0 \quad \text { as } \quad m \rightarrow \infty .
$$

We set

$$
\begin{aligned}
\tilde{B} & :=\{\tilde{v}(\varphi(z)) f(\varphi(z)) ; f \in C, z \in \mathbf{D}\} \\
B_{m} & :=\left\{\tilde{v}(\varphi(z)) \cdot C_{m} f(\varphi(z)) ; f \in C, z \in \mathbf{D}\right\}, \quad m \in \mathbf{N},
\end{aligned}
$$

and we take

$$
B:=\tilde{B} \cup \bigcup_{m=0}^{\infty} B_{m} .
$$

We claim that $B$ is bounded in $X$. Indeed, if $p \in c s(X)$, there is a constant $M_{p}$ such that

$$
\sup _{z \in \mathbf{D}} \tilde{v}(z) p(f(z)) \leq M_{p}
$$


for each $f \in C$. Moreover, for each $m \in \mathbf{N}, z \in \mathbf{D}$, and $f \in C$, we can apply [1, Lemma 1.1] to get

$$
\sup _{z \in \mathbf{D}} \tilde{v}(\varphi(z)) p\left(C_{m} f(\varphi(z))\right) \leq \sup _{z \in \mathbf{D}} \tilde{v}(\varphi(z)) p(f(\varphi(z))) \leq M_{p}
$$

Hence $p(\xi) \leq M_{p}$ for each $\xi \in B$.

Fix $\varepsilon>0$. Select $\left.r_{0} \in\right] 0,1\left[\right.$ for $B, q, \frac{\varepsilon}{2}>0$ as in $(b)$. To prove (3.4) we split the estimate as follows

$$
\sup _{z \in \mathbf{D}} w(z) q\left(W_{\psi, \varphi} f(z)-W_{\psi, \varphi} C_{m} f(z)\right) \leq\left(s_{1}\right)+\left(s_{2}\right),
$$

where

and

$$
\left(s_{1}\right)=\sup _{|\varphi(z)|>r_{0}} w(z) q\left(W_{\psi, \varphi} f(z)-W_{\psi, \varphi} C_{m} f(z)\right)
$$

$$
\left(s_{2}\right)=\sup _{|\varphi(z)| \leq r_{0}} w(z) q\left(W_{\psi, \varphi} f(z)-W_{\psi, \varphi} C_{m} f(z)\right) .
$$

We analyze first $\left(s_{1}\right)$. We use the simple estimate

$$
w(z) q\left(W_{\psi, \varphi} f(z)-W_{\psi, \varphi} C_{m} f(z)\right) \leq w(z) q\left(W_{\psi, \varphi} f(z)\right)+w(z) q\left(W_{\psi, \varphi} C_{m} f(z)\right) .
$$

For $|\varphi(z)|>r_{0}$, we have

$$
\begin{aligned}
w(z) q\left(W_{\psi, \varphi} f(z)\right) & =w(z) q(\psi(z)[f(\varphi(z))]) \\
& =\frac{w(z)}{\tilde{v}(\varphi(z))} q(\psi(z)[\tilde{v}(\varphi(z)) f(\varphi(z))]) \leq \frac{\varepsilon}{2}
\end{aligned}
$$

since $\tilde{v}(\varphi(z)) f(\varphi(z)) \in B$. Now, if $|\varphi(z)|>r_{0}$,

$$
w(z) q\left(W_{\psi, \varphi} C_{m} f(z)\right) \leq \frac{w(z)}{\tilde{v}(\varphi(z))} q\left(\psi(z)\left[\tilde{v}(\varphi(z)) \cdot C_{m} f(\varphi(z))\right]\right) \leq \frac{\varepsilon}{2} .
$$

Therefore, $\left(s_{1}\right) \leq \varepsilon$ for each $m$. Next, we study $\left(s_{2}\right)$. Observe first, that for $f \in C$ we have at least formally,

$$
W_{\psi, \varphi} f(z)-W_{\psi, \varphi} C_{m} f(z)=\frac{1}{m+1}\left(\sum_{k=0}^{m}\left(\sum_{\ell=k+1}^{\infty} \varphi(z)^{\ell} \psi(z)\left[\frac{f^{(\ell)}(0)}{\ell !}\right]\right)\right) .
$$

Then

$$
\left(s_{2}\right) \leq \sup _{|\varphi(z)| \leq r_{0}} w(z) \frac{1}{m+1} \sum_{k=0}^{m}\left(\sum_{\ell=k+1}^{\infty}|\varphi(z)|^{\ell} q\left(T_{\psi}\left(\frac{f^{(\ell)}(0)}{\ell !}\right) z\right)\right) .
$$

By assumption $T_{\psi}$ is Montel, hence it also is continuous. There is $p \in c s(X)$ such that the Cauchy Integral Formula for derivatives yields

$$
w(z) q\left(T_{\psi}\left(\frac{f^{(\ell)}(0)}{\ell !}\right) z\right) \leq p\left(\frac{f^{(\ell)}(0)}{\ell !}\right) \leq \frac{1}{r^{\ell}} \max _{|\zeta|=r} p(f(\zeta)) \leq \frac{1}{r^{\ell} v(r)} \sup _{\zeta \in \mathbf{D}} v(\zeta) p(f(\zeta)) .
$$

Then, if $r>r_{0}$, as $|\varphi(z)| \leq r_{0}$ in $\left(s_{2}\right)$ we obtain

$$
\begin{aligned}
\left(s_{2}\right) & \leq \sup _{z \in \mathbf{D}} v(z) p(f(z))\left(\frac{1}{m+1} \sum_{k=0}^{m}\left(\sum_{\ell=k+1}^{\infty}\left(\frac{r_{0}}{r}\right)^{\ell} \frac{1}{v(r)}\right)\right) \\
& =\sup _{z \in \mathbf{D}} v(z) p(f(z)) \frac{1}{v(r)}\left[\sum_{\ell=0}^{\infty}\left(\frac{r_{0}}{r}\right)^{\ell}-\frac{1}{m+1} \sum_{k=0}^{m} S_{k}\right],
\end{aligned}
$$


where $S_{k}=\sum_{\ell=0}^{k}\left(\frac{r_{0}}{r}\right)^{\ell}$ is the $k$-th partial sum of the convergent geometric series $\sum_{\ell=0}^{\infty}\left(\frac{r_{0}}{r}\right)^{\ell}$, which shows that $\left(s_{2}\right)$ tends to 0 as $m$ tends to infinity, and (3.3) is proved.

Now, we suppose that $W_{\psi, \varphi}$ is Montel. Since $T_{\psi}$ inherits the properties of $W_{\psi, \varphi}$, we obtain that $T_{\psi}$ is Montel. To prove the condition (b) we argue by contradiction. If (b) does not hold then there exist an absolutely convex bounded set $B$ in $X$ and $q \in \operatorname{cs}(Y)$ such that for all $n \in \mathbf{N}$ there exists $z_{n} \in \mathbf{D}$ with $\left|\varphi\left(z_{n}\right)\right|>1-\frac{1}{n}$ and there exists $x_{n} \in B$ such that the following inequality holds:

$$
\frac{w\left(z_{n}\right)}{\tilde{v}\left(\varphi\left(z_{n}\right)\right)} q\left(\psi\left(z_{n}\right)\left[x_{n}\right]\right)>1
$$

The set

$$
M:=\left\{f \in H_{v}^{\infty}(\mathbf{D}, X): v(z) f(z) \in B \text { for all } z \in \mathbf{D}\right\}
$$

is bounded in $H_{v}^{\infty}(\mathbf{D}, X)$. Since $W_{\psi, \varphi}$ is Montel, $W_{\psi, \varphi} M$ is relatively compact in $H_{w}^{\infty}(\mathbf{D}, Y)$. For each $n \in \mathbf{N}$, select functions $f_{n} \in H(\mathbf{D})$ verifying

$$
\left|f_{n}\right| \leq \frac{1}{v} \quad \text { and } \quad f_{n}\left(\varphi\left(z_{n}\right)\right)=\frac{1}{\tilde{v}\left(\varphi\left(z_{n}\right)\right)}
$$

and we define

$$
h_{n}(z)=z^{n} f_{n}(z) x_{n}, \quad z \in \mathbf{D} .
$$

Clearly, $h_{n} \in H_{v}^{\infty}(\mathbf{D}, X)$ and $v(z) h_{n}(z) \in B$ for all $z \in \mathbf{D}$. Hence $\left(h_{n}\right)_{n=1}^{\infty} \subseteq M$. Furthermore, we claim that $\left(h_{n}\right)_{n=1}^{\infty}$ converges to 0 in $H(\mathbf{D}, X)$ for the open-compact topology as $n$ tends to infinity. In order to prove this, fix $p \in c s(X)$ and $\left.r_{0} \in\right] 0,1[$. We have

$$
\sup _{|z| \leq r_{0}} p\left(h_{n}(z)\right)=\sup _{|z| \leq r_{0}}|z|^{n} p\left(f_{n}(z) x_{n}\right) \leq r_{0}^{n} \frac{1}{\tilde{v}\left(r_{0}\right)} \sup _{z \in \mathbf{D}} \tilde{v}(z) p\left(f_{n}(z) x_{n}\right) \leq C r_{0}^{n} \frac{1}{\tilde{v}\left(r_{0}\right)}
$$

for some constant $C>0$. Therefore $\lim _{n \rightarrow \infty} \sup _{|z| \leq r_{0}} p\left(h_{n}(z)\right)=0$.

Since $W_{\psi, \varphi}: H(\mathbf{D}, X) \longrightarrow H(\mathbf{D}, Y)$ is continuous by Proposition 5 , the sequence $\left(W_{\psi, \varphi} h_{n}\right)_{n=1}^{\infty}$ converges to 0 in $H(\mathbf{D}, Y)$ for the open-compact topology. Moreover $W_{\psi, \varphi} M$ is relatively compact in $H_{w}^{\infty}(\mathbf{D}, Y)$ and $\left(W_{\psi, \varphi} h_{n}\right)_{n=1}^{\infty} \subseteq W_{\psi, \varphi} M$. Hence $\left(W_{\psi, \varphi} h_{n}\right)_{n=1}^{\infty}$ converges to 0 in $H_{w}^{\infty}(\mathbf{D}, Y)$. But, if we take $q \in \operatorname{cs}(Y)$ and $\left(z_{n}\right)_{n=1}^{\infty}$ satisfying (3.5), there exists $n_{0}$ such that, for $n \geq n_{0}$,

$$
\begin{aligned}
w\left(z_{n}\right) q\left(\left(W_{\psi, \varphi} h_{n}\right)\left(z_{n}\right)\right) & =w\left(z_{n}\right) q\left(\psi\left(z_{n}\right)\left[h_{n}\left(\varphi\left(z_{n}\right)\right)\right]\right) \\
& =w\left(z_{n}\right) q\left(\psi\left(z_{n}\right)\left[\varphi\left(z_{n}\right)^{n} f_{n}\left(\varphi\left(z_{n}\right)\right) x_{n}\right]\right) \\
& =w\left(z_{n}\right) q\left(\varphi\left(z_{n}\right)^{n} f_{n}\left(\varphi\left(z_{n}\right)\right) \psi\left(z_{n}\right)\left[x_{n}\right]\right) \\
& =w\left(z_{n}\right)\left|\varphi\left(z_{n}\right)\right|^{n} \frac{1}{\tilde{v}\left(\varphi\left(z_{n}\right)\right)} q\left(\psi\left(z_{n}\right)\left[x_{n}\right]\right)>\left(1-\frac{1}{n}\right)^{n}>\frac{1}{e}
\end{aligned}
$$

which contradicts the fact that $\left(W_{\psi, \varphi} h_{n}\right)_{n=1}^{\infty}$ converges to 0 in $H_{w}^{\infty}(\mathbf{D}, Y)$. The proof is complete. [8].

The following consequences should be compared with some results presented in

Corollary 15. Let $L: X \rightarrow Y$ be a non-zero linear and continuous operator and $\psi: \mathbf{D} \rightarrow L_{b}(X, Y)$ is the constant operator-valued analytic function equal to $L \neq 0$ for all $z \in \mathbf{D}$. The following holds: 
(i) $W_{\psi, \varphi}: H_{v}^{\infty}(\mathbf{D}, X) \rightarrow H_{w}^{\infty}(\mathbf{D}, Y)$ is continuous if and only if the composition operator $C_{\varphi}: H_{v}^{\infty}(\mathbf{D}) \rightarrow H_{w}^{\infty}(\mathbf{D})$ is continuous.

(ii) $W_{\psi, \varphi}: H_{v}^{\infty}(\mathbf{D}, X) \rightarrow H_{w}^{\infty}(\mathbf{D}, Y)$ is bounded if and only if

(a) $L$ is bounded, and

(b) $C_{\varphi}: H_{v}^{\infty}(\mathbf{D}) \rightarrow H_{w}^{\infty}(\mathbf{D})$ is continuous.

(iii) $W_{\psi, \varphi}: H_{v}^{\infty}(\mathbf{D}, X) \rightarrow H_{w}^{\infty}(\mathbf{D}, Y)$ is Montel if and only if

(a) $L$ is Montel, and

(b) $C_{\varphi}: H_{v}^{\infty}(\mathbf{D}) \rightarrow H_{w}^{\infty}(\mathbf{D})$ is compact.

As a concrete example, if $v(z)=(1-|z|)^{\alpha}, w(z)=(1-|z|)^{\beta}, \varphi(z)=z$ and $\phi(z)=$ $L \in L(X, Y), L \neq 0$ Montel, for each $z \in \mathbf{D}$, then the operator $W_{\psi, \varphi}^{\infty}: H_{v}^{\infty}(\mathbf{D}, X) \rightarrow$ $H_{w}^{\infty}(\mathbf{D}, Y)$ is Montel if $0<\alpha<\beta$, but it is continuous not Montel if $0<\alpha=\beta$.

Complete characterizations of the continuity and compactness of composition operators $C_{\varphi}: H_{v}^{\infty}(\mathbf{D}) \rightarrow H_{w}^{\infty}(\mathbf{D})$ in terms of the weights $v$ and $w$ and the symbol $\varphi$ were obtained [7], [4] and [11].

The following result gives a sufficient condition to obtain a version of the equivalence between $(b)$ and $(c)$ in Theorem 9 for the case of weighted spaces. Such a result should be compared with [16, Theorem 4.4], that treats the case of Banach valued analytic functions. In order to get the equivalence, it is necessary to put some restrictions on $\psi$; see Theorem 22 .

For a weight function $v$, we define the following space:

$$
H_{v}^{0}(\mathbf{D}, X)=\left\{f \in H_{v}^{\infty}(\mathbf{D}, X): \lim _{|z| \rightarrow 1-} v(z) p(f(z))=0 \text { for all } p \in c s(X)\right\} .
$$

In particular, if $v \equiv 1$, then $H_{v}^{0}(\mathbf{D}, X)=\{0\}$.

Theorem 16. Let $X, Y$ be complete barrelled locally convex spaces. If $\psi \in$ $H_{w}^{0}\left(\mathbf{D}, L_{b}(X, Y)\right)$, then the following assertions are equivalent:

(a) The operator $T_{\psi}: X \rightarrow H_{w}^{\infty}(\mathbf{D}, Y)$ is Montel.

(b) $\psi(z): X \rightarrow Y$ is Montel for all $z \in \mathbf{D}$.

Proof. Condition (a) implies condition (b) as a consequence of Remark 4 (ii). We prove then that (b) implies (a). Since $\psi$ is analytic, we can write

$$
\psi(z)=\sum_{\ell=0}^{\infty} A_{\ell} z^{\ell}, \quad A_{\ell} \in L(X, Y) .
$$

By the Cauchy Integral Formula for the coefficients $A_{\ell}$, it follows that $A_{\ell}$ is a Montel operator from $X$ to $Y$. See, e.g., [22] for the integration of continuous functions with values in a complete locally convex space. We denote by $C_{m} \psi$ the Cesàro sums of the Taylor polynomials associated to $\psi$. We check that the corresponding auxiliary operators $T_{C_{m} \psi}$ are Montel operators. To do this, it suffices to show that if $g: \mathbf{D} \rightarrow L(X, Y)$ is an operator-valued monomial $g(z)=A z^{\ell}$ where $A: X \rightarrow Y$ is a Montel operator, then the corresponding auxiliary operator $T_{g}: X \rightarrow H_{w}^{\infty}(\mathbf{D}, Y)$ is Montel. Fix a bounded set $B$ in $X$. By assumption, $A(B)$ is relatively compact in $Y$. The set

$$
T_{g}(B)=\left\{A(b) z^{\ell}: b \in B\right\}
$$

is relatively compact in $H_{w}^{\infty}(\mathbf{D}, Y)$. Indeed, the linear operator

$$
S: Y \longrightarrow H_{w}^{\infty}(\mathbf{D}, Y), \quad y \longmapsto\left[z \mapsto y z^{\ell}\right]
$$

is also continuous and satisfies $T_{g}(B)=S(A(B))$. 
Since the space of Montel operators is closed in the space of all linear and continuous operators $([15,42.1(3)])$, the proof is complete if we show that $T_{C_{m} \psi}$ converges to $T_{\psi}$ in $L\left(X, H_{w}^{\infty}(\mathbf{D}, Y)\right)$. Let $B$ be a bounded set in $X$ and $q$ a continuous seminorm on $Y$. We want to estimate

$$
\sup _{b \in B} \sup _{z \in \mathbf{D}} w(z) q\left(\left(C_{m} \psi(z)-\psi(z)\right)(b)\right) .
$$

Since $\psi \in H_{w}^{0}\left(\mathbf{D}, L_{b}(X, Y)\right)$, for each $\varepsilon>0$ there exists $R_{0}>0$ such that for all $|z| \geq R_{0}$ we have

$$
\sup _{b \in B} w(z) q(\psi(z)(b))<\varepsilon / 3
$$

For each $b \in B$, the function $z \mapsto \psi(z)(b)$ is in $H(\mathbf{D}, Y)$. Therefore, for each $b \in B$ and $m \in \mathbf{N}$, we can apply [1, Lemma 1.1] and Hahn-Banach theorem to conclude

$$
w(z) q\left(C_{m} \psi(z)(b)\right) \leq \max _{|\zeta|=|z|} w(\zeta) q(\psi(\zeta)(b)) .
$$

Thus, for $|z|>R_{0}, b \in B$ and $m \in \mathbf{N}$,

$$
w(z) q(\psi(z)(b))<\varepsilon / 3 \text { and } w(z) q\left(C_{m} \psi(z)(b)\right)<\varepsilon / 3 .
$$

Applying again that $\psi \in H_{w}^{\infty}(\mathbf{D}, L(X, Y))$, the Cesàro sums of the Taylor polynomials $C_{m} \psi$ of $\psi$ converge to $\psi$ in the topology of $H(\mathbf{D}, L(X, Y))$. In particular, there exist $m_{0} \in \mathbf{N}$ such that, for all $m \geq m_{0}$, we have

$$
\sup _{|z| \leq R_{0}} \sup _{b \in B} w(z) q\left(\psi(z)(b)-C_{m} \psi(z)(b)\right)<\varepsilon / 3 .
$$

Therefore, for every $\varepsilon>0, B$ bounded in $X$ and $q$ continuous seminorm on $Y$, there is $m_{0}$ (and $R_{0}>0$ ), such that for $m \geq m_{0}$, we obtain

$$
\begin{aligned}
& \sup _{b \in B} \sup _{z \in \mathbf{D}} w(z) q\left(T_{\psi}(b)(z)-T_{C_{m} \psi}(b)(z)\right) \\
& =\sup _{b \in B} \sup _{z \in \mathbf{D}} w(z) q\left(\psi(z)(b)-C_{m} \psi(z)(b)\right) \\
& \leq \sup _{b \in B}\left\{\sup _{|z| \geq R_{0}} w(z) q(\psi(z)(b))+\sup _{|z| \geq R_{0}} w(z) q\left(C_{m} \psi(z)(b)\right)\right. \\
& \left.\quad+\sup _{|z| \leq R_{0}} w(z) q\left(\psi(z)(b)-C_{m} \psi(z)(b)\right)\right\}<\varepsilon .
\end{aligned}
$$

\section{Analytic dependence of diagonal operators in values of Köthe spaces}

In this section we restrict our attention to operator weighted composition operators between spaces of analytic functions on the unit disc with values in a Köthe echelon space. The image $\psi(z)$ is a diagonal operator for each $z \in \mathbf{D}$.

We refer the reader to [21] and [3] for a detailed study of Köthe echelon spaces. We recall here a few definitions. A matrix $A=\left(a_{n}(i)\right)_{i, n \in \mathbf{N}}$ of non-negative numbers if called a Köthe matrix if it satisfies the following conditions:

(1) For each $i \in \mathbf{N}$ there is $n \in \mathbf{N}$ with $a_{n}(i)>0$.

(2) $a_{n}(i) \leq a_{n+1}(i)$ for all $i, n \in \mathbf{N}$.

For $1 \leq p<\infty$ we define

$$
\lambda_{p}(A):=\left\{x \in \mathbf{C}^{\mathbf{N}}: q_{n}(x):=\left(\sum_{i=1}^{\infty}\left|x_{i} a_{n}(i)\right|^{p}\right)^{1 / p}<\infty \text { for all } n \in \mathbf{N}\right\}
$$


and for $p=\infty$ and $p=0$ :

$$
\begin{aligned}
& \lambda_{\infty}(A):=\left\{x \in \mathbf{C}^{\mathbf{N}}: q_{n}(x):=\sup _{i \in \mathbf{N}}\left|x_{i}\right| a_{n}(i)<\infty \text { for all } n \in \mathbf{N}\right\}, \\
& \lambda_{0}(A):=\left\{x \in \lambda_{\infty}(A): \lim _{i \rightarrow \infty} a_{n}(i) x_{i}=0 \text { for all } n \in \mathbf{N}\right\} .
\end{aligned}
$$

The spaces $\lambda_{p}(A), 1 \leq p \leq \infty$ and $p=0$ are Fréchet spaces with a fundamental sequence of seminorms $\left(q_{n}\right)_{n \in \mathbf{N}}$. From now on, we write $\lambda_{p}=\lambda_{p}(A)$ and we fix a weight function $w$ on $\mathbf{D}$.

Proposition 17. Let $\left(f_{i}\right)_{i \in \mathbf{N}}$ be a sequence in $H(\mathbf{D})$, bounded for the compactopen topology. For $1 \leq p \leq \infty$ or $p=0$, the diagonal operator-valued function defined

$$
\psi: \mathbf{D} \longrightarrow L_{b}\left(\lambda_{p}, \lambda_{p}\right), \quad z \longmapsto \psi(z)(x)=\left(f_{i}(z) x_{i}\right)_{i \in \mathbf{N}}
$$

is well-defined, continuous and analytic.

Proof. It is easy to see that $\psi(z)$ is continuous for each $z \in \mathbf{D}$, since $\left(f_{i}\right)_{i \in \mathbf{N}}$ is bounded in the compact sets of $\mathbf{D}$. Moreover, $\psi: \mathbf{D} \rightarrow L_{b}\left(\lambda_{p}, \lambda_{p}\right)$ is locally bounded on D. Indeed, the set $\psi(\{z \in \mathbf{D}:|z| \leq R\})$ is bounded in $L_{b}\left(\lambda_{p}, \lambda_{p}\right)$ for each $R \in] 0,1\left[\right.$. To check this, fix a bounded set $B \subset \lambda_{p}$ and $n \in \mathbf{N}$, and estimate as follows, for $1 \leq p<\infty$,

$$
\sup _{|z| \leq R} \sup _{x \in B} q_{n}(\psi(z)(x))=\sup _{|z| \leq R} \sup _{x \in B}\left(\sum_{i=1}^{\infty}\left|a_{n}(i) f_{i}(z) x_{i}\right|^{p}\right)^{1 / p} \leq C_{R} \sup _{x \in B} q_{n}(x)<\infty,
$$

for some constant $C_{R}>0$ that depends on $R$ and the bounded sequence $\left(f_{i}\right)_{i \in \mathbf{N}}$. The cases $p=0$ or $p=\infty$ are easier.

To prove that $\psi$ is analytic, by a result of Grosse-Erdmann [13, Theorem 1], we have only to show that $\gamma \circ \psi: \mathbf{D} \rightarrow \mathbf{C}$ is analytic for each $\gamma$ in a weak ${ }^{*}$-dense set $G \subset L_{b}\left(\lambda_{p}, \lambda_{p}\right)^{\prime}$. First we consider the case $p \neq \infty$. Given $u \in\left(\lambda_{p}\right)^{\prime}, y \in \lambda_{p}$ and $T \in L_{b}\left(\lambda_{p}, \lambda_{p}\right)$ we write

$$
(u \otimes y)(T):=\langle T(y), u\rangle=u(T(y)),
$$

which is a continuous linear functional $u \otimes y$ on $L_{b}\left(\lambda_{p}, \lambda_{p}\right)$. Define

$$
G:=\operatorname{span}\left\{u \otimes y: u \in\left(\lambda_{p}\right)^{\prime}, y \in \lambda_{p}\right\} .
$$

It is easy to see that $G$ is a weak*-dense subset of $L_{b}\left(\lambda_{p}, \lambda_{p}\right)^{\prime}$. It remains to show that $(u \otimes y) \circ \psi$ is analytic on $\mathbf{D}$ for each $u \in\left(\lambda_{p}\right)^{\prime}$ and $y \in \lambda_{p}$. If $u \in\left(\lambda_{p}\right)^{\prime}$ we can identify it with a sequence $\left(u_{i}\right)_{i \in \mathbf{N}}$ such that for each $y=\left(y_{i}\right)_{i \in \mathbf{N}} \in \lambda_{p}$, we have $\sum_{i=1}^{\infty}\left|u_{i} y_{i}\right|<\infty$ by the Köthe duality (see [21, Chapter 27]). Therefore

$$
((u \otimes y) \circ \psi)(z)=(u \otimes y)(\psi(z))=u\left(\left(f_{i}(z) y_{i}\right)=\sum_{i=1}^{\infty} u_{i} y_{i} f_{i}(z)\right.
$$

defines an analytic function on $\mathbf{D}$.

If $p=\infty$, we repeat the proof with $G:=\operatorname{span}\left\{u \otimes y: u \in\left(\lambda_{0}\right)^{\prime}, y \in \lambda_{\infty}\right\}$.

The following essentially well-known lemma follows from the characterization of bounded sets in a Köthe echelon space in [3]. 
Lemma 18. If $\left(\gamma_{i}\right)_{i \in \mathbf{N}}$ is a sequence in $c_{0}$, the linear and continuous diagonal operator

$$
L: \lambda_{p} \longrightarrow \lambda_{p}, \quad\left(x_{i}\right)_{i \in \mathbf{N}} \longmapsto\left(\gamma_{i} x_{i}\right)_{i \in \mathbf{N}}
$$

is a Montel operator.

As a consequence, if $\psi$ is the map of Proposition 17 , then $\psi(z): \lambda_{p} \rightarrow \lambda_{p}$ is a Montel operator for each $z \in \mathbf{D}$ in case the sequence of analytic functions $\left(f_{i}\right)_{i \in \mathbf{N}}$ tends to 0 for the compact-open topology.

An application of Theorem 5, Proposition 17, and Theorem 9 to the present situation yields the following result.

Corollary 19. (i) If $\left(f_{i}\right)_{i \in \mathbf{N}}$ is a bounded sequence in $H(\mathbf{D})$ for the compactopen topology, then $W_{\psi, \varphi}: H\left(\mathbf{D}, \lambda_{p}\right) \rightarrow H\left(\mathbf{D}, \lambda_{p}\right)$ is continuous.

(ii) If $\left(f_{i}\right)_{i \in \mathbf{N}}$ tends to 0 for the compact-open topology, then

(a) $\psi(z): \lambda_{p} \rightarrow \lambda_{p}$ is a Montel operator for every $z \in \mathbf{D}$,

(b) $W_{\psi, \varphi}: H\left(\mathbf{D}, \lambda_{p}\right) \rightarrow H\left(\mathbf{D}, \lambda_{p}\right)$ is Montel,

(c) $T_{\psi}: \lambda_{p} \rightarrow H\left(\mathbf{D}, \lambda_{p}\right)$ is Montel.

We now treat the case of weighted spaces of analytic functions with values in a Köthe echelon space. To do this, we fix a sequence $\left(f_{i}\right)_{i \in \mathbf{N}}$ in $H_{w}^{\infty}(\mathbf{D})$ such that $\left\|f_{i}\right\|_{w}:=\sup _{z \in \mathbf{D}} w(z)\left|f_{i}(z)\right| \leq 1$. Clearly, $\left(f_{i}\right)_{i \in \mathbf{N}}$ is bounded in $H(\mathbf{D})$ for the compact open topology, hence the operator $\psi: \mathbf{D} \longrightarrow L_{b}\left(\lambda_{p}, \lambda_{p}\right), z \mapsto \psi(z)(x)=\left(f_{i}(z) x_{i}\right)_{i \in \mathbf{N}}$ is analytic by Proposition 17.

Our purpose now is to study the associated operator

$$
T_{\psi}: \lambda_{p} \longrightarrow H_{w}^{\infty}\left(\mathbf{D}, \lambda_{p}\right), \quad T_{\psi}(x)[z]=\left(f_{i}(z) x_{i}\right)_{i \in \mathbf{N}}
$$

We will show in Theorem 22 that $T_{\psi}$ need not to be Montel even if $\psi(z)$ is Montel for each $z \in \mathbf{D}$, thus showing that the conclusion of Theorem 16 does not hold without the assumption $\psi \in H_{w}^{0}\left(\mathbf{D}, L_{b}(X, Y)\right)$, and that the equivalence in Theorem 9 fails in the weighted case. An example in the Banach valued case is given in [16, Example 4.1] for $f_{i}(z)=z^{i}, i \in \mathbf{N}, w(z)=1$ and $\lambda_{1}=\ell_{1}$.

Lemma 20. If $\left(f_{i}\right)_{i \in \mathbf{N}}$ in $H_{w}^{\infty}(\mathbf{D})$ satisfies $\left\|f_{i}\right\|_{w} \leq 1$, then the operator

$$
T_{\psi}: \lambda_{p} \longrightarrow H_{w}^{\infty}\left(\mathbf{D}, \lambda_{p}\right), \quad T_{\psi}(x)[z]=\left(f_{i}(z) x_{i}\right)_{i \in \mathbf{N}}
$$

is well defined and continuous.

Proof. For every $n \in \mathbf{N}$, we have

$$
\sup _{z \in \mathbf{D}} w(z) q_{n}\left(T_{\psi}(x)[z]\right)=\sup _{z \in \mathbf{D}} w(z)\left(\sum_{i=1}^{\infty}\left|a_{n}(i) f_{i}(z) x_{i}\right|^{p}\right)^{1 / p} \leq q_{n}(x),
$$

which proves the assertion.

Lemma 21. There is a sequence $\left(f_{i}\right)_{i \in \mathbf{N}}$ in $H_{w}^{\infty}(\mathbf{D})$ such that

(a) there is $\varepsilon>0$ satisfying $\varepsilon<\left\|f_{i}\right\|_{w} \leq 1$ for every $i \in \mathbf{N}$,

(b) $\left(f_{i}\right)_{i \in \mathbf{N}}$ tends to 0 for the compact-open topology.

Proof. Since $H_{w}^{\infty}(\mathbf{D})$ is infinite dimensional in the present setting, we can find a sequence $\left(g_{i}\right)_{i \in \mathbf{N}}$ in $H_{w}^{\infty}(\mathbf{D})$ with $\left\|g_{i}\right\|_{w} \leq 1$ for every $i \in \mathbf{N}$ which cannot have a Cauchy subsequence. Passing to a subsequence if necessary, we find $\varepsilon_{0}>0$ with 
$\left\|g_{i}-g_{j}\right\|_{w} \geq \varepsilon_{0}$ for each $i, j \in \mathbf{N}$. The unit ball of $H_{w}(\mathbf{D})$ is compact for compactopen topology, therefore there exists a subsequence $\left(h_{j}\right)_{j \in \mathbf{N}}$ of $\left(g_{i}\right)_{i \in \mathbf{N}}$, convergent to $h_{0}$ in $H(\mathbf{D})$. We put $f_{j}:=\frac{1}{2}\left(h_{j}-h_{0}\right), j \in \mathbf{N}$. Clearly each $f_{j}$ belongs to the unit ball of $H_{w}^{\infty}(\mathbf{D}),\left\|f_{j}-f_{k}\right\|_{w}=\frac{1}{2}\left\|h_{j}-h_{k}\right\|_{w} \geq \varepsilon_{0} / 2$ and $\left(f_{j}\right)_{j \in \mathbf{N}}$ tends to 0 for the compact-open topology.

Theorem 22. Let $\left(f_{i}\right)_{i \in \mathbf{N}} \subset H_{w}^{\infty}(\mathbf{D})$ satisfy $\left\|f_{i}\right\|_{w} \leq 1$ for all $i \in \mathbf{N}$. Then

(a) If $\lambda_{p}$ is Montel, then $T_{\psi}$ is a Montel operator.

(b) If $\left(f_{i}\right)_{i \in \mathbf{N}}$ tends to 0 for the compact-open topology, then $\psi(z): \lambda_{p} \rightarrow \lambda_{p}$ is a Montel operator for every $z \in \mathbf{D}$.

(c) Assume that there is $\varepsilon>0$ satisfying $\varepsilon<\left\|f_{i}\right\|_{w}$ for every $i \in \mathbf{N}$. If $\lambda_{p}$ is not Montel, then $T_{\psi}$ is not a Montel operator.

Proof. (a) If $\lambda_{p}$ is Montel, $T_{\psi}$ is Montel, because it is continuous by Lemma 20 and the bounded subsets of $\lambda_{p}$ are relatively compact.

(b) follows from Corollary 19 (ii).

(c) If $\lambda_{p}$ is not Montel, there is an infinite subset $J$ of natural numbers and $m \in \mathbf{N}$ such that for all $n \in \mathbf{N}$ there is $c_{n}>0$ satisfying $a_{n}(j) \leq c_{n} a_{m}(j)$ for each $j \in J$ (see [21, Chapter 27]). Define

$$
B:=\left\{\frac{e_{j}}{a_{m}(j)}: j \in J\right\},
$$

where $\left(e_{j}\right)_{j \in \mathbf{N}}$ is the canonical basis in $\lambda_{p}$. The set $B$ is bounded in $\lambda_{p}$ since, for every $n \in \mathbf{N}$,

$$
\sup _{j \in \mathbf{N}} q_{n}\left(\frac{e_{j}}{a_{m}(j)}\right)=\sup _{j \in \mathbf{N}} \frac{a_{n}(j)}{a_{m}(j)} \leq c_{n} .
$$

If the operator $T_{\psi}$ were Montel, the set $\left(T_{\psi}\left(\frac{e_{j}}{a_{m}(j)}\right)\right)_{j \in \mathbf{N}}$ would have a convergent subsequence in $H_{w}^{\infty}\left(\mathbf{D}, \lambda_{p}\right)$. But, for $i \neq j$, we have

$$
\begin{aligned}
& \sup _{z \in \mathbf{D}} w(z) q_{m}\left(T_{\psi}\left(\frac{e_{j}}{a_{m}(j)}\right)(z)-T_{\psi}\left(\frac{e_{i}}{a_{m}(i)}\right)(z)\right) \\
& =\sup _{z \in \mathbf{D}} w(z) q_{m}\left(\psi(z)\left(\frac{e_{j}}{a_{m}(j)}\right)-\psi(z)\left(\frac{e_{i}}{a_{m}(i)}\right)\right) \\
& =\sup _{z \in \mathbf{D}} w(z) q_{m}\left(\frac{f_{j}(z)}{a_{m}(j)} \cdot e_{j}-\frac{f_{i}(z)}{a_{m}(i)} \cdot e_{i}\right) \\
& =\sup _{z \in \mathbf{D}} w(z)\left(\left|f_{i}(z)\right|^{p}+\left|f_{j}(z)\right|^{p}\right)^{1 / p} \\
& \geq \max \left(\sup _{z \in \mathbf{D}} w(z)\left|f_{i}(z)\right|, \sup _{z \in \mathbf{D}} w(z)\left|f_{j}(z)\right|\right) \geq \varepsilon .
\end{aligned}
$$

Propositions 12 and 13 yield the following consequences in our present setting.

Corollary 23. Let $v$ be a weight, $\varphi(z)=z$ and $\psi: \mathbf{D} \rightarrow L_{b}\left(\lambda_{p}, \lambda_{p}\right), z \mapsto$ $\psi(z)(x)=\left(f_{i}(z) x_{i}\right)_{i \in \mathbf{N}}$, with $\left\|f_{i}\right\|_{v} \leq 1$. Then

(a) $W_{\psi, i d}: H_{v}^{\infty}\left(\mathbf{D}, \lambda_{p}\right) \rightarrow H_{v}^{\infty}\left(\mathbf{D}, \lambda_{p}\right)$ is continuous if and only if for each $n \in \mathbf{N}$ there is $m \in \mathbf{N}$ with $m \geq n$ such that

$$
\left\{\frac{a_{n}(i)}{a_{m}(i)} f_{i}: i \in \mathbf{N}\right\}
$$

is bounded in $H^{\infty}(\mathbf{D})$. 
(b) $W_{\psi, i d}: H_{v}^{\infty}\left(\mathbf{D}, \lambda_{p}\right) \rightarrow H_{v}^{\infty}\left(\mathbf{D}, \lambda_{p}\right)$ is bounded if and only if there is $m \in \mathbf{N}$ such that, for every $n \in \mathbf{N}$, the set

$$
\left\{\frac{a_{n}(i)}{a_{m}(i)} f_{i}: i \in \mathbf{N}\right\}
$$

is bounded in $H^{\infty}(\mathbf{D})$.

We finish this section with an example in the setting of Köthe echelon spaces which shows that a result like Theorem 9 is not true in general if we replace being a Montel operator by being bounded.

Proposition 24. There exists a Köthe matrix $A$ such that

$$
\psi(z): \lambda_{1}(A) \longrightarrow \lambda_{1}(A), \quad x \longmapsto \psi(z)(x)=\left(z^{i} x_{i}\right)_{i \in \mathbf{N}}
$$

is bounded for each $z \in \mathbf{D}$, but the corresponding auxiliary operator $T_{\psi}: \lambda_{1}(A) \rightarrow$ $H\left(\mathbf{D}, \lambda_{1}(A)\right)$ is not bounded.

Proof. Set $R_{1}:=1$ and $R_{n}:=\frac{2 n+1}{n+1}$ for $n \geq 2$ and define a Köthe matrix $A$ by $a_{n}(i)=\left(R_{n}\right)^{i}$, for each $n, i \in \mathbf{N}$. We show that the operator $\psi(z)(x)=\left(z^{i} x_{i}\right)_{i \in \mathbf{N}}$ is bounded for each $z \in \mathbf{D}$. Given $z$, select the smallest $m \in \mathbf{N}$ such that $\frac{m-1}{m} \leq|z|<$ $\frac{m}{m+1}$. For every $n \in \mathbf{N}$,

$$
R_{n}|z|<2|z|<\frac{2 m}{m+1}<R_{m},
$$

hence $a_{n}(i)|z|^{i} \leq a_{m}(i)$ for this $m$ and every $n \in \mathbf{N}$. This implies that $\psi(z)$ is bounded.

Now suppose that the operator $T_{\psi}: \lambda_{1} \rightarrow H\left(\mathbf{D}, \lambda_{1}\right)$ is bounded. There is $m \in \mathbf{N}$ such that the image $T_{\psi}\left(U_{m}\right)$ of the neighborhood of 0

$$
U_{m}:=\left\{x \in \lambda_{1}: \sum_{i=1}^{\infty} a_{m}(i)\left|x_{i}\right| \leq 1\right\}
$$

is bounded. Therefore, there is $m \in \mathbf{N}$ such that for every $0<R<1$ and $n \in \mathbf{N}$, there is $C>0$ such that, for each $x \in U_{m}$,

$$
\sup _{|z| \leq R} \sum_{i=1}^{\infty} R_{n}^{i}|z|^{i}\left|x_{i}\right| \leq C .
$$

In particular, there is $m$ such that for every $0<R<1$ and $n \in \mathbf{N}$, there is $C>0$ satisfying, for each $i \in \mathbf{N}$,

$$
\sup _{|z| \leq R} R_{n}^{i}|z|^{i} \leq C R_{m}^{i}
$$

Then, there is $m \in \mathbf{N}$ such that for each $n \in \mathbf{N}$ and $0<R<1$, we have $R_{n} R \leq R_{m}$. Letting $n \rightarrow \infty$, there is $m \in \mathbf{N}$ such that $2 R \leq 1+\frac{m}{m+1}$ for all $0<R<1$, a contradiction.

\section{References}

[1] Bierstedt, K. D., J. Bonet, and A. Galbis: Weighted spaces of holomorphic functions on balanced domains. - Michigan Math. J. 40:2, 1993, 271-297.

[2] Bierstedt, K. D., J. Bonet, and J. Taskinen: Associated weights and spaces of holomorphic functions. - Studia Math. 127, 1998, 137-168. 
[3] Bierstedt, K. D., R. Meise, and W. Summers: Köthe sets and Köthe sequence spaces. North-Holland Mathematics Studies 71, 1982, 27-91.

[4] Bonet, J., P. Domański, and M. Lindström: Essential norm and weak compactness of composition operators on weighed Banach spaces of analytic functions. - Canad. Math. Bull. 42, 1999, 139-148.

[5] Bonet, J., P. Domański, and M. Lindström: Pointwise multiplication operators on weighted Banach spaces of analytic functions. - Studia Math. 137, 1999, 177-194.

[6] Bonet, J., P. Domański, and M. Lindström: Weakly compact composition operators on analytic vector-valued functions spaces. - Ann. Acad. Sci. Fenn. Math. 26, 2001, 233-248.

[7] Bonet, J., P. Domański, M. Lindström, and J. Taskinen: Composition operators between weighted Banach spaces of analytic functions. - J. Austral. Math. Soc. Ser. A 64, 1998, 101-118.

[8] Bonet, J., and M. Friz: Weakly compact composition operators on locally convex spaces. Math. Nachr. 245, 2002, 26-44.

[9] Bonet, J., M. Lindström, and E. Wolf: Isometric weighted composition operators on weighted Banach spaces of type $H^{\infty}$. - Proc. Amer. Math. Soc. 136:12, 2008, 4267-4273.

[10] Cichon, K., and K. Seip: Weighted holomorphic spaces with trivial closed range multiplication operators. - Proc. Amer. Math. Soc. 131, 2003, 201-207.

[11] Contreras, M., and A. G. Hernández-Díaz: Weighted composition operators in weighted Banach spaces of analytic functions. - J. Aust. Math. Soc. Ser. A 69, 2000, 41-60.

[12] Cowen, C., and B. MacCluer: Composition operators on spaces of analytic functions. CRC Press, 1995.

[13] Grosse-Erdmann, K.-G.: A weak criterion for vector-valued holomorphic functions. - Math. Proc. Cambridge Philos. Soc. 136, 2004, 399-411.

[14] Jarchow, H.: Locally convex spaces. - Teubner, Stuttgart, 1981.

[15] Köтнe, G.: Topological vector spaces. II. - Grundlehren Math. Wiss. 237, Springer-Verlag, New York-Berlin, 1979.

[16] Laitila, J., and H.-O. Tylli: Operator-weighted composition operators on vector-valued analytic function spaces. - Illinois Math. J. 53, 2009, 1019-1032.

[17] Laitila, J., H.-O. TylLi, and M. WANG: Composition operators from weak to strong spaces of vector-valued analytic functions. - J. Operator Theory 62:2, 2009, 281-295.

[18] Lindström, M., and E. Wolf: Essential norm of the difference of weighted composition operators. - Monatsh. Math. 153:2, 2008, 133-143.

[19] Lusky, W.: On the isomorphism classes of weighted spaces of harmonic and holomorphic functions. - Studia Math. 175, 2006, 19-45.

[20] Manhas, J. S.: Weighted composition operators on weighted spaces of vector-valued analytic functions. - J. Korean Math. Soc. 45, 2008, 1203-1220.

[21] Meise, R., and D. Vogt: Introduction to functional analysis. - Oxford Univ. Press, 1997.

[22] Rudin, W.: Functional analysis. - International Series in Pure and Applied Mathematics, McGraw-Hill, New York, 1991.

[23] Ruess, W.: Compactness and collective compactness in spaces of compact operators. - J. Math. Anal. Appl. 84, 1981, 400-417.

[24] Shapiro, J. H.: Composition operators and classical function theory. - Springer-Verlag, 1993.

[25] Vukotic, D.: Pointwise multiplication operators between Bergman spaces on simply connected domains. - Indiana Univ. Math. J. 48, 1999, 793-803. 